\title{
Ceftazidime/avibactam and ceftolozane/tazobactam for the treatment of extensively drug-resistant Pseudomonas aeruginosa post-neurosurgical infections: three cases and a review of the literature
}

\author{
Marianna Meschiari ${ }^{1}$ (D - lacopo Franconi ${ }^{2} \cdot$ Erica Bacca $^{2} \cdot$ Vincenzo Bianco $^{3} \cdot$ Gabriella Orlando $^{1}$. Gianluca Cuomo ${ }^{1}$. \\ Andrea Bedini ${ }^{1}$. Cristina Mussini ${ }^{2}$
}

Received: 24 July 2020 / Accepted: 8 October 2020 / Published online: 19 October 2020

(c) Springer-Verlag GmbH Germany, part of Springer Nature 2020

\begin{abstract}
Purpose Post-neurosurgical infection caused by extensively drug resistant Pseudomonas aeruginosa (XDR-PA) are becoming a matter of great concern due to limited therapeutic options. Although not approved for these indications, the new BetaLactam-BetaLactamase Inhibitor combinations (BLBLIs) could represent a valid salvage treatment. We describe one nosocomial meningitis and two cervical osteomyelitis due to an XDR-PA who were treated with ceftazidime/avibactam $(\mathrm{CZA})$ and ceftolozane/tazobactam $(\mathrm{C} / \mathrm{T})$ and review the literature.

Methods The first and the third patients developed an osteomyelitis following cervical stabilization surgery due to an XDRPA. Although the first patient started treatment with a high dose of $C / T$, resistance to $C / T$ occurred, so therapy was switched to CZA plus aztreonam. The third patient switched to aztreonam plus CZA due to development of acute kidney injury during therapy with colistin. The second patient had an XDR-PA meningitis following the insertion of an external ventricular catheter and he was treated with $\mathrm{C} / \mathrm{T}$ plus meropenem and amikacin.

Results All three cases reported were successfully conservatively treated thanks to the use of the new BLBLIs with different combinations. Only few experiences demonstrated an equally favorable outcome: one patient treated with $\mathrm{C} / \mathrm{T}$ plus fosfomycin for otogenic meningitis caused by an XDR-PA and another case of XDR-PA post-surgical meningitis with CZA in combination with colistin. Finally, the combination of CZA plus aztreonam has proven to be effective on XDR-PA only in limited mostly in vitro studies.

Conclusion These recently developed antibiotics, C/T and CZA are promising and complementary therapy options against post-neurosurgical hard-to-treat $\mathrm{P}$. aeruginosa infections. Further prospective real-life studies are required to validate these findings in this special setting.
\end{abstract}

Keywords Extensively drug resistant Pseudomonas aeruginosa $\cdot$ Meningitis $\cdot$ Cervical osteomyelitis $\cdot$ Ceftazidime/ avibactam $\cdot$ Ceftolozane/tazobactam

Marianna Meschiari

marianna.meschiari1209@gmail.com;

meschiari.marianna@policlinico.mo.it

Cristina Mussini

cristina.mussini@unimore.it

1 Infectious Diseases Unit, Azienda Ospedaliero-Universitaria Policlinico and University of Modena and Reggio Emilia, Modena, Italy

2 Department of Infectious Diseases, University of Modena and Reggio Emilia, Modena, Italy

3 Infectious Diseases Clinic, Università degli Studi Federico II, Naples, Italy

\section{Introduction}

Extensively drug resistant Pseudomonas aeruginosa (XDRPA) is an emerging pathogen associated with high mortality ranging from 30 to $50 \%$ due to poor therapeutic options [1]. Data regarding infections caused by XDR-PA are limited to case reports and series of urinary tract infections and ventilator-associated pneumonia [2-4]. Even fewer reports are available regarding central nervous system (CNS) infections and cervical osteomyelitis [5-7]. Due to these limited data, the best therapeutic options for XDR-PA infection in 
this specific setting have yet to be defined [8,9]. Although not (yet) FDA approved for these indications, the new BetaLactam-BetaLactamase Inhibitor combinations (BLBLIs) could represent a valid salvage treatment for patients with XDR-PA. We describe three patients with XDR-PA postneurosurgical infection who were treated with new BLBLIs in our clinical practice in 2018 and review the literature.

\section{Case 1}

A 66-year-old man, with no previous medical history, was referred to our emergency department for tetraparesis following cervical trauma. The spinal cord computed tomography (CT) scan revealed a compound fracture of the vertebral body of C6, associated with a disjointed fracture of the C7 transverse epiphysis to the right, and with rupture of the intervertebral disk at C6-C7. The patient underwent immediate cervical stabilization, and after $48 \mathrm{~h}$ an external fixation device was placed. On the 24th day of hospitalization, the patient developed severe acute obstructive respiratory failure, fever $\left(39^{\circ} \mathrm{C}\right)$ and leukocytosis $\left(15,000 \mathrm{WBC} / \mathrm{mm}^{3}\right.$ (neutrophils 85\%). The chest radiograph showed increased density of the right basal lobe, suggestive of pneumonia. In addition, pus drained from a fixator pin. Empirical antibiotic therapy with piperacillin/tazobactam (TZP) plus linezolid was started. Cultures from pus and bronchoalveolar-lavage (BAL) showed growth of a carbapenem-resistant PA. Clinical and microbiological findings confirmed cervical vertebral osteomyelitis. Table 1 panel P1.1 shows the antibiogram of the isolated pathogen. TZP was suspended and ciprofloxacin $400 \mathrm{mg}$ three times daily was initiated. On the 34th day the external fixator was removed and an internal osteosynthetic fixation was performed. On the 45th day purulent material drained again from the wound, and the patient had a fever $\left(38.5^{\circ} \mathrm{C}\right)$, a rise in C-reactive protein (CRP) $(18.5 \mathrm{mg} /$ $\mathrm{dL}$, normal limit $5 \mathrm{mg} / \mathrm{dL}$ ) and leukocytosis. A CT scan of the neck and thorax showed a $4 \times 1.5 \mathrm{~cm}$ collection in the right paratracheal area and two confluent collections behind the spinous processes of $\mathrm{C} 5$ and $\mathrm{C} 6,6 \mathrm{~cm}$ in diameter. No surgical reintervention was deemed possible. New cultures from aspirate of the wound showed only growth of PA, now also resistant to ciprofloxacin (MIC $>4 \mathrm{mcg} / \mathrm{ml}$ ). Antibiotic therapy was changed to ceftolozane/tazobactam (C/T) $3 \mathrm{~g}$ three times daily plus amikacin $15 \mathrm{mg} / \mathrm{kg}$ once daily. On the 55th day of hospitalization, 7 days after the last antibiotic escalation, the patient's clinical condition worsened with purulent secretion from the wound. Cultures of the aspirate showed only growth of XDR-PA resistant to C/T (Table 1 P1.2). Antibiotic therapy was switched to ceftazidime-avibactam (CZA) $2.5 \mathrm{~g}$ four times daily in extended infusion plus aztreonam $2 \mathrm{~g}$ four times daily for 6 weeks. At the end of treatment on the 100th day, magnetic resonance imaging (MRI) showed complete bone consolidation and the patient was discharged.

\section{Case 2}

A 57-year-old woman was admitted to the Intensive Care Unit (ICU) due to left cerebral brain spontaneous intraparenchymal hemorrhage. She presented with vomiting, bradycardia, anisocoria, bilateral mydriasis and impaired consciousness requiring intubation and artificial ventilation. As a brain CT scan reported hydrocephalic dilatation of the ventricular system, an external ventricular drain (EVD) was inserted. After 20 days of clinical improvement, the 21 th day the patient developed fever $\left(39^{\circ} \mathrm{C}\right)$ leukocytosis $\left(20,000 \mathrm{WBC} / \mathrm{mm}^{3}, 90 \%\right.$ neutrophils), and was comatose requiring a new cycle of artificial ventilation. On suspicion of a hospital-acquired meningitis (HAM), empirical antibiotic treatment consisting of continuousinfusion vancomycin and extended-infusion meropenem was initiated. Cerebrospinal fluid (CSF) obtained from the EVD contained 15.000 cells $/ \mathrm{mm}^{3}$ and grew cefotaximesusceptible Klebsiella pneumoniae. Antibiotic therapy was
Table 1 Antimicrobial susceptibility patterns of XDR-PA isolates

\begin{tabular}{lllllllllll}
\hline Patient number & Isolate number & AMK & FEP & MEM & CIP & TZP & CST & CZA & C/T & ATM \\
\hline P1 & 1.1 & 2 & $>32$ & $>32$ & 0,5 & $>32$ & $\leq 1$ & 4 & 2 & 12 \\
& 1.2 & $>16$ & $>32$ & $>32$ & $>4$ & $>128$ & $>8$ & 8 & $>16$ & 12 \\
P2 & 2.1 & 4 & 16 & 32 & 1 & 16 & $\leq 1$ & 8 & 2 & \\
P3 & 3.1 & $\leq 2$ & 16 & 4 & $>2$ & $>32$ & $\leq 1$ & 4 & $\leq 1$ & 32 \\
& 3.2 & 2 & $>16$ & 64 & $>2$ & $>32$ & 1 & 16 & 4 & 32 \\
\hline
\end{tabular}

AMK amikacin, FEP cefepime, MEM meropenem, CIP ciprofloxacin, CST colistin, TZP piperacillin-tazobactam, $C Z A$ ceftazidime-avibactam, $C / T$ ceftolozane-tazobactam, $A T M$ aztreonam; from https://aac.asm. org/content/abbreviations-and-conventions

Susceptibility patterns were expressed by the different values of minimum inhibitory concentration (MIC), determined by broth microdilution using the Micronaut S MDR MRGN-Screening 3 panel (MERLIN Diagnostika GmbH). MICs were interpreted taking as reference the EUCAST 2018 clinical breakpoint values (susceptibility of PA to C/T and CZA for MIC values of $\leq 4$ and $\leq 8 \mathrm{mcg} / \mathrm{ml}$, respectively) 
deescalated to ceftriaxone $2 \mathrm{~g}$ twice daily. After 10 days of antibiotic therapy the whole EVD system was changed. On the 44th day the patient developed again fever $\left(39^{\circ} \mathrm{C}\right)$, leukocytosis, and impaired consciousness. Presuming a recrudescence of HAM, antibiotic therapy was escalated to meropenem $2 \mathrm{~g}$ four times daily along with vancomycin $30 \mathrm{mg} / \mathrm{kg}$. CSF cultures via EVD showed growth of XDR-PA. The susceptibility is shown in Table $1 \mathrm{P} 2.1$. The EVD was removed and another reinserted after several days; antibiotic therapy was escalated to $\mathrm{C} / \mathrm{T} 3 \mathrm{~g}$ three times daily and meropenem $2 \mathrm{~g}$ four times daily, both by extended infusion and associated with amikacin $15 \mathrm{mg} /$ $\mathrm{kg}$ once daily. Three weeks after antibiotic escalation, a ventriculoperitoneal shunt was implanted. One week later, after obtaining a negative CSF culture, the antibiotic treatment was stopped and the patient showed no evidence of CSF infection thereafter.

\section{Case 3}

A 29-year-old man was referred to our hospital ninety days after surgical vertebral fixation performed elsewhere for a cervical trauma C6-C7. The patient presented with fever $\left(39^{\circ} \mathrm{C}\right)$ leukocytosis $\left(23,000 \mathrm{WBC} / \mathrm{mm}^{3}\right)$ and a rise in CRP $(30 \mathrm{mg} / \mathrm{dl})$. A CT scan showed presence of four small lamellar bone formations anterior-laterally to the vertebral body of D1 on the left, compatible with dislocation of fragments of osteosynthetic material and a fluid collection anterior of the vertebral bodies of C5-C6-C7, adjacent to the esophagus. The presumed diagnosis was cervical osteomyelitis with mediastinitis due to esophageal fistulization. Empirical antibiotic treatment with TZP plus linezolid was started the same day. Surgical debridement and closure of a skin-esophageal fistula were performed the next day. The intraoperative cultures yielded XDR-PA plus Carbapenemase-producing Klebsiella pneumoniae (KPC). Table 1 P3.1 shows the susceptibility pattern. Antibiotic therapy was escalated on the 6th day to extendedinfusion CZA $2.5 \mathrm{~g}$ three times daily plus colistin $9 \mathrm{MU}$ once daily. On the 11th day the patient developed acute renal failure so CST was stopped and aztreonam $2 \mathrm{~g}$ four times per day was added. A repeat CT scan showed that the prevertebral fluid collection adjacent to the posterior cervical esophagus extended caudally to the posteriorsuperior mediastinum. A new surgical debridement was performed on the 20th day. The therapy was continued for a total of 8 weeks and a CT scan showed complete resolution of the picture on 60th day of hospitalization. The rectal swab performed for routinely screening at the end of treatment yielded XDR PA with acquired resistance to $\mathrm{CZA}(\mathrm{MIC}=16 \mathrm{mcg} / \mathrm{ml})($ Table $1 \mathrm{P} 3.2)$.

\section{Discussion}

XDR-PA healthcare associated infections are of great concern due to limited therapeutic options and high attributable mortality [2]. To date, the BLBLIs C/T and CZA have been approved for complicated urinary tract infection and complicated intra-abdominal infection $[10,11]$. Recently, CZA was approved for the treatment of hospital-acquired pneumonia and, in Europe, also for Gram-negative infections with limited treatment options [11]. Only few data are available on post-neurosurgical osteomyelitis and central nervous system (CNS) infections [5-7]. However, from these preliminary results on hard-to-treat infections, these 2 antibiotics appear to be a promising therapeutic option.

Our patients 1 and 3 had cervical osteomyelitis in the presence of internal osteosynthetic fixation material that could not be safely removed, and was successfully treated with CZA associated with aztreonam. The reasons for the use of CZA were resistance to C/T in patient 1 and superinfection with KPC in patient 3, while we added ATM considering the paper by Davido B et al. which demonstrated the superiority of the combination of ATM and CAZ/AVI due to synergistic activity on an AmpC-hyperproducing strain of Pseudomonas aeruginosa susceptible only to amikacin and colistin (MIC, $0.25 \mathrm{mg} / \mathrm{l}$ )" [13].

To the best of our knowledge, these are the first confirmed cases of cervical osteomyelitis caused by XDR-PA. It is well recognized that this rare site of bone infection has limited surgical options due to high incidence of neurological deficits that occur as a result of direct spinal cord compression by epidural abscess, segmental deformity and instability [14]. So, on one hand we were obliged to use the best available and powerful antibiotic treatment, on the other the strain had acquired resistance to $\mathrm{C} / \mathrm{T}$ thus limiting treatment options. Indeed, although we have used in both cases a high-dose regimen of C/T [10] to improve bone penetration, resistance to $\mathrm{C} / \mathrm{T}$ occurred during treatment. In case 3, combination therapy with colistin had to be discontinued early due to acute kidney injury. However, while colistin and aminoglycoside could be an effective option in this specific setting, these drugs have also important limitations: rapid emergence of resistance especially when used in monotherapy, pharmacokinetic restrictions due to inadequate drug concentrations in target action sites and several toxicities, especially nephrotoxicity [15]. This experience of the management of XDR-PA cervical vertebral osteomyelitis shows the success of combination antibiotic therapy and sample-based follow-up in order to prevent further development of antibiotic resistance.

The second case describes an XDR-PA CNS infection treated with a combination therapy of high- dose $\mathrm{C} / \mathrm{T}$, 
amikacin and meropenem. To the best of our knowledge, just one other experience was reported showing an equally favorable outcome with high-dose $\mathrm{C} / \mathrm{T}$ plus fosfomycin for treatment of otogenic meningitis caused by XDR-PA [6]. The role of combination therapy remains to be clarified, as well as the optimal dose to obtain sufficient penetration into the CNS. Extended infusion of beta-lactam antibiotics, in particular meropenem, are currently recommended for meningitis to optimize CSF penetration. [16]. A later published Monte Carlo simulation seems to predict a better probability of target attainment increasing dosages in patients with Klebsiella pneumoniae carbapenemase (KPC)-producing Klebsiella pneumoniae (KPC-Kp) infections with MIC of up to $64 \mathrm{mg} /$ liter [17].

A study on blood-brain barrier penetration among critically ill patients with an infected EVD is still ongoing [12]. Sterilization of CSF in MDR bacterial infections has been shown with intrathecal and intravenous colistin $[8,9]$. However, this strategy is not easy to perform for reasons of toxicity, allergy and limited neurosurgical expertise with the drug, or not feasible in case of early EVD removal [9].

In conclusion, we think that either high-dose $\mathrm{C} / \mathrm{T}$ or $\mathrm{CZA}$ alone or in combination therapy might be valuable and complementary options for post-neurosurgical infections due to XDR-PA. Further prospective real-life studies are required to validate these findings in this special setting.

Acknowledgements We thank Inge C. Gyssens for critical review of the manuscript.

Funding The study received no specific grants from any funding agency in the public, commercial, or not-for-profit sector.

Availability of data and materials Data from the current study are available from the corresponding author on reasonable request.

\section{Compliance with ethical standards}

Conflict of interest The authors declare that they have no conflict of interest.

Ethical approval This study was reviewed and approved by the University of Modena and Reggio Emilia local institutional Ethical Committee (Comitato Etico Provinciale di Modena). The study was conducted in accordance with the provisions of the Declaration of Helsinki and Good Clinical Practice guidelines.

Consent to participate All involved persons gave their informed verbal witnessed consent.

Consent for publication Consent for publication was obtained from all participants to the study. Data were published anonymously.

\section{References}

1. Magiorakos AP, Srinivasan A, Carey RB, Carmeli Y, Falagas ME, Giske CG, et al. Multidrug-resistant, extensively drugresistant and pandrug-resistant bacteria: an international expert proposal for interim standard definitions for acquired resistance. Clin Microbiol Infect. 2012;18:268-81.

2. Munita JM, Aitken SL, Miller WR, Perez F, Rosa R, Shimose LA, et al. Multicenter evaluation of ceftolozane/tazobactam for serious infections caused by carbapenem-resistant Pseudomonas aeruginosa. Clin Infect Dis. 2017;65:158-61.

3. Rodríguez-Núñez O, Ripa M, Morata L, de la Calle C, Cardozo C, Fehér C, Pellicé M, Valcárcel A, Puerta-Alcalde P, Marco F, García-Vidal C, del Río A, Soriano A. J Martínez-Martínez Evaluation of ceftazidime/avibactam for serious infections due to multidrug-resistant and extensively drug-resistant Pseudomonas aeruginosa. J Glob Antimicrob Resist. 2018;15:136-9.

4. Bassetti M, Castaldo N, Cattelan A, Mussini C, Righi E, Tascini $\mathrm{C}$, et al. Ceftolozane/tazobactam for the treatment of serious Pseudomonas aeruginosa infections: a multicentre nationwide clinical experience. Int J Antimicrob Agents. 2019;53:408-15.

5. Dietl B, Sánchez I, Arcenillas P, Cuchi E, Gómez L, González de Molina FJ, Boix-Palop L, Nicolás J, Calbo E. Ceftolozane/ tazobactam in the treatment of osteomyelitis and skin and softtissue infections due to extensively drug-resistant Pseudomonas aeruginosa: clinical and microbiological outcomes. Int J Antimicrobial Agents. 2018;51:498-5027.

6. Frattaria Antonella, Savini Vincenzo, Polilli Ennio, Cibelli Donatella, Talamazzi Silvia, Bosco Donatella, Consorte Augusta, Fazii Paolo, Parruti Giustino. Ceftolozane-tazobactam and Fosfomycin for rescue treatment of otogenous meningitis caused by XDR Pseudomonas aeruginosa: case report and review of the literature. ID Cases. 2018;31:00451.

7. Xipell M, Bodro M, Marco F, Losno RA, Cardozo C, Soriano A. Clinical experience with ceftazidime/avibactam in patients with severe infections, including meningitis and lung abscesses, caused by extensively drug-resistant Pseudomonas aeruginosa. Int J Antimicrob Agents. 2017;49:259-68.

8. Tunkel Allan R, Hasbun Rodrigo, Bhimraj Adarsh, Byers Karin, Kaplan Sheldon L, Michael Scheld W, van de Beek Diederik, Bleck Thomas P, Garton Hugh JL, Zunt Joseph R. Infectious Diseases Society of America's Clinical Practice Guidelines for healthcare-associated ventriculitis and meningitis. Clin Infect Dis. 2017;64:34-65.

9. Karvouniaris M, Brotis AG, Tsiamalou P, et al. The role of intraventricular antibiotics in the treatment of nosocomial ventriculitis/meningitis from gram-negative pathogens: a systematic review and meta-analysis. World Neurosurg. 2018;120:637-50.

10. Solomkin J, Hershberger E, Miller B, et al. Ceftolozane/tazobactam plus metronidazole for complicated intra-abdominal infections in an era of multidrug resistance: results from a randomized, double-blind, phase 3 trial (ASPECT-cIAI). Clin Infect Dis. 2015;60:1462-71.

11. Das S, Li J, Riccobene T, Carrothers TJ, Newell P, Melnick D, Critchley IA, Stone GG, Nichols WW. Dose selection and validation for ceftazidime-avibactam in adults with complicated intra-abdominal infections, complicated urinary tract infections, and nosocomial pneumonia. Antimicrob Agents Chemother. 2019;63:02187-18.

12. Roberts JA. Ceftolozane-tazobactam pharmacokinetics in infected critically ill patients with an indwelling External Ventricular Drain, PhD. ClinicalTrials.gov identifier (NCT number): NCT03309657. Australia: Royal Brisbane and Women's Hospital, University of Brisbane; 2018. Accessed 15 Feb 2018. 
13. Davido B, Fellous L, Lawrence C, Maxime V, Rottman M, Dinh A. Ceftazidime-avibactam and aztreonam, an interesting strategy to overcome $\beta$-lactam resistance conferred by metallo- $\beta$ lactamases in Enterobacteriaceae and Pseudomonas aeruginosa. Antimicrob Agents Chemother. 2017;61:e01008-17.

14. Meher SK, Jain H, Tripathy LN, Basu S. Chronic Pseudomonas aeruginosa cervical osteomyelitis. J Craniovertebr Junction Spine. 2016;7:276-8. https://doi.org/10.4103/0974-8237.193264.

15. Ordooei Javan A, Shokouhi S, Sahraei Z. A review on colistin nephrotoxicity. Eur J Clin Pharmacol. 2015;71:801-10. https:// doi.org/10.1007/s00228-015-1865-4 (Epub 2015 May 27).

16. Lu C, Zhang Y, Chen M, Zhong P, Chen Y, Yu J, Wu X, Wu J, Zhang J. Population pharmacokinetics and dosing regimen optimization of meropenem in cerebrospinal fluid and plasma in patients with meningitis after neurosurgery. Antimicrob Agents Chemother. 2016;60:6619-25. https://doi.org/10.1128/aac.00997 $-16$.

17. Cojutti P, Sartor A, Righi E, Scarparo C, Bassetti M, Pea F. Population pharmacokinetics of high-dose continuous- infusion meropenem and considerations for use in the treatment of infections due to KPC-producing Klebsiella pneumoniae. Antimicrob Agents Chemother. 2017;61:e00794-17. https://doi.org/10.1128/ aac.00794-17. 